\title{
Zur Rechtsstellung von Familienmitgliedern aus Drittstaaten im Lichte der Kernbereichs-Rechtsprechung des EuGH
}

\section{Von Daniel Thym, Konstanz*}

In der Kontroverse um die Unionsbürgerschaft steht derzeit ein Thema im Vordergrund: die Rechtsstellung von drittstaatsangehörigen Familienmitgliedern. Dieser neue Aufmerksamkeitsschwerpunkt ist kein Zufall, sondern entspringt dem überstaatlichen Charakter der Unionsbürgerschaft, weil die Freizügigkeit durch den Familiennachzug gefördert werden soll. Diese abgeleiteten Rechte der Familienmitglieder deuteten der EU-Gesetzgeber und der EuGH immer wieder expansiv - zuletzt infolge des Ruiz Zambrano-Urteils. Freilich stößt diese Expansion der Unionsbürgerrechte an Grenzen, weil der EU-Bürgerstatus nicht alles regeln soll und kann. Aus diesem Grund akzentuiert der Beitrag einen alternativen verfassungsrechtlichen Bezugsrahmen: das Migrationsrecht im Raum der Freiheit, der Sicherheit und des Rechts. Es zeigt sich, dass jenseits der Unionsbürgerschaft kein rechtliches Niemandsland herrscht. Die Menschenrechte leiten den Familiennachzug dort, wo die Unionsbürgerschaft nicht hinreicht.

\section{Vorbemerkung}

Die Zeiten ändern sich und mit ihnen die Aufmerksamkeitsschwerpunkte. Dies gilt auch für die Diskussion um die Unionsbürgerschaft. Über ein Jahrzehnt standen die Sozialleistungen im Brennpunkt. Doch hier wurden die zentralen Weichen inzwischen gestellt. In der Freizügigkeits-Richtlinie billigte der EU-Gesetzgeber den weiten Zugriff des EuGH auf den Gleichheitssatz seit Martinez Sala und umgekehrt wich der EuGH in Förster einen Schritt zurück und akzeptierte eine Ausnahme, auf die der Gesetzgeber bestanden hatte. ${ }^{1}$ Seither diskutieren wir über Einzelfragen, die eine große rechtspraktische Bedeutung besitzen. ${ }^{2}$ Die verfassungsrechtlichen Grundsätze beim Zugang zu Sozialleistungen sind geklärt.

Indes verlagerte sich der Streit auf ein neues Themenfeld. Wenn man die kontroversen EuGH-Urteile des letzten Jahrzehnts zur Unionsbürgerschaft gedanklich Revue passieren lässt, sticht ein gemeinsamer Nenner neben Sozialleistungen hervor: der Rechtsstatus von drittstaatsangehörigen Familienmitgliedern. Ruiz Zambrano und Dereci sind die prominentesten Beispiele, aber die Reihe an Urteilen, auf

\footnotetext{
* Prof. Dr. Daniel Thym, LL.M. ist Inhaber des Lehrstuhls für öffentliches Recht, Europa- und Völkerrecht sowie Ko-Direktor des Forschungszentrums Ausländer- und Asylrecht (FZAA) an der Universität Konstanz.

1 S. M. Dougan, The Bubble that Burst, in: Adams et al. (Hrsg.), Judging Europe's Judges. The Legitimacy of the Case Law of the European Court of Justice Examined, 2013, S. 127 (145-153) sowie D. Thym, Hindernisse auf dem Weg zur „wahrhaftigen“" Unionsbürgerschaft, ZEuS 2012, S. 501 (507-514).

2 Etwa den zulässigen Ausschluss von arbeitsuchenden Unionsbürgern von „Hartz IV“-Leistungen (hierzu T. Kingreen, Staatsangehörigkeit als Differenzierungskriterium im Sozialleistungsrecht, SGb 2013, S. 132) sowie die Grenzen des Aufenthaltsrechts von nichterwerbstätigen Unionsbürgern bei Sozialleistungsbezug (hierzu $D$. Thym, Sozialleistungen für und Aufenthalt von nichterwerbstätigen Unionsbürgern, NZS 2014, S. 81-90).
} 
denen dieser Beitrag beruht, ist länger. Die meisten Europarechtler kennen Entscheidungen wie Carpenter, Baumbast, Akrich, Zhu \& Chen, Eind, Metock, Ibrahim, McCarthy, Iida, $O \& S$ oder Ymeraga und Alopka. All diese Urteile betreffen verschiedene Fragen des Primär- und Sekundärrechts, besitzen jedoch eine $\mathrm{Ge}-$ meinsamkeit: Es geht um Familienangehörige von Unionsbürgern - das Thema dieses Beitrags.

Dieses soll in drei weiteren Schritten dargelegt werden. Zuerst verdeutlicht der Blick zurück die Besonderheit des EU-Ansatzes, wenn der EU-Gesetzgeber sowie der EuGH den Familiennachzug nahezu unbedingt fördern (II.). Auf dieser Grundlage werden die dogmatischen Einzelheiten der Rechtsstellung in verschiedenen Konstellationen ausgeführt, mit einem Schwerpunkt auf den jüngeren Entscheidungen über das abgeleitete Aufenthaltsrecht von Familienangehörigen aufgrund des Kernbereichsschutzes nach dem Ruiz Zambrano-Urteil (III.). Hierbei wird deutlich, dass verbleibende Unschärfen aus der Gemengelage von Unionsbürgerschaft und Migrationsrecht folgen. Letzteres begründet einen alternativen verfassungsrechtlichen Bezugsrahmen. Es zeigt sich, dass jenseits der Unionsbürgerschaft kein rechtliches Niemandsland herrscht. Die Menschenrechte regeln den Familiennachzug dort, wo die Unionsbürgerschaft nicht hinreicht (IV.).

\section{Familienzusammenführung als Selbstzweck}

Es wäre falsch, die Geschichte der Unionsbürgerrechte mit dem Vertrag von Maastricht oder dem Urteil Martínez Sala beginnen zu lassen. Die EU-Integration beinhaltete von Anfang an ein überschießendes, bürgerschaftliches Element, das über die Wirtschaftsgemeinschaft hinausreichte: die Vorschriften zur Arbeitnehmerfreizügigkeit. Freilich bezog der EWG-Vertrag diese einzig auf die Erwerbstätigen selbst. ${ }^{3}$ Von Familienangehörigen ist im Vertragstext bis heute keine Rede. Wenn diese in der Gesetzgebung und der Rechtsprechung der folgenden Jahrzehnte dennoch eine privilegierte Rechtsstellung erhielten, verdeutlicht dies umso mehr, wie großzügig der EU-Ansatz in der Anfangsdekade ist. Beim Familiennachzug wird ganz ähnlich wie beim Zugang zu Sozialleistungen - deutlich, dass Arbeitnehmer keineswegs nur als wirtschaftliche Produktionsfaktoren gefördert wurden, sondern ihre Lebensbedingungen durch Migration verbessern sollten. ${ }^{4}$ In der Europäischen Wirtschaftsgemeinschaft war die Marktbürgerschaft bis zu einem gewissen Grad immer auch Sozialbürgerschaft. ${ }^{5}$

3 S. Art. 48 EWGV (1957) sowie den heutigen Art. 45 AEUV; dass die Bestimmungen nicht explizit auf Staatsangehörige der Mitgliedstaaten beschränkt wurden, lag am Streit um die Rechtsstellung von Franzosen aus Algerien sowie Sorgen der Bundesrepublik um die Rechtsstellung von DDR-Bürgern; vgl. S. Goedings, Labor Migration in an Integrating Europe, 2005, S. 128-135.

4 Anschaulich die Erwgr. 4 und 6 VO (EU) Nr. 492/2011 (ABI. EU 2011 L 141/1) als Nachfolgerin der früheren VO (EWG) Nr. 1612/68 (ABI. EG 1968 L 257/2).

5 Weiterführend $T$. Kingreen, Die Universalisierung sozialer Rechte im europäischen Gemeinschaftsrecht, EuRBeiheft 1-2007, S. 43; L. Azoulai, La Citoyenneté européenne, un statut d'intégration sociale, in: Piris u.a. (Hrsg.), Chemins d'Europe. Melanges en I'honneur de Jean Paul Jacqué, 2010, S. 1 und D. Kochenov, The Citizenship Paradigm, C.Y.E.L.S. 15 (2013), Abschn. 2. 
Was geschah im Einzelnen? Lange vor den ersten EuGH-Urteilen musste der EUGesetzgeber die schlichten Vorgaben des EU-Vertrags im Wege der Sekundärrechtsetzung konkretisieren. Hierbei erlangten in den 1960er-Jahren zuerst die Kernfamilie und später auch die erweiterte Großfamilie ein Nachzugsrecht, obgleich hiervon im Vertragstext keine Rede war. ${ }^{6}$ Diese Gewährleistung eines nahezu unbedingten Familiennachzugs vollzog sich vergleichsweise geräuschlos, wobei speziell die Kommission und Italien für eine großzügige Regelung stritten, die die anderen Mitgliedstaaten nach anfänglichem Widerstand in Bezug auf Großfamilien akzeptierten. ${ }^{7}$ Bis heute gilt: Die gesamte Familie von Arbeitnehmern unter Einschluss der Eltern besitzt ein gleichsam automatisches Nachzugsrecht.

Einzig eine Bedingung wurde damals gegen den Widerstand der Kommission im Sekundärrecht niedergelegt: ausreichend Wohnraum musste vorhanden sein. ${ }^{8}$ Dies Kriterium entsprang der Wohnungsnot der Nachkriegszeit infolge der Kriegszerstörungen und wurde zwischenzeitlich aufgegeben. ${ }^{9}$ Hinzu kommen die vertragsunmittelbaren Ausnahmen für Gründe der öffentlichen Ordnung, Sicherheit und Gesundheit, die traditionell restriktiv zu handhaben sind. ${ }^{10}$ Weitere Ausnahmen wurden nicht vorgesehen und sind demgemäß bis heute verboten. Insbesondere müssen Arbeitnehmer für sich und ihre Familie bis heute nicht die finanzielle Sicherung des Lebensunterhalts nachweisen. ${ }^{11}$ Die Sicherung des Lebensunterhalts von Arbeitnehmern wurde in den 1960er-Jahren wohl nicht als mögliches Problem betrachtet, weil man davon ausging, dass ein Arbeiter eine Familie zu ernähren vermag. ${ }^{12}$ In gleicher Weise dürfen die Mitgliedstaaten von mobilen Unionsbürgern keine gesellschaftliche Integration, etwa durch den Erwerb von Sprachkenntnissen, verlangen. Debatten um sprachliche Integrationserwartungen sind ein vergleichs-

6 Art. 11 VO (EWG) Nr. 15/61 (ABl. EG 196I Nr. 57, S. 1073) betraf noch einzig die Kernfamilie, nachdem die Benelux-Staaten und Deutschland dem vermeintlichen Nachzug von mediterranen GroBfamilien („Clans") widersprochen hatten; großzügiger später Art. 17 Abs. 1 VO (EWG) Nr. $38 / 64$ (ABl. EG 1964 Nr. 62, S. 965), der mit Art. 10 VO (EWG) Nr. 1612/68 (Fn. 4) und Art. 7 Abs. 1 Buchst. d i.V.m. Art. 2 Nr. 2 RL 2004/38/EG (ABI. EU 2004 L 158/77) übereinstimmt.

7 Zum Verhandlungsverlauf siehe $S$. Goedings, Labor Migration (Fn. 3), S. 163-166 und $220 \mathrm{f}$

8 S. Art. 11 Abs. 3 VO (EWG) Nr. 15/61 (Fn. 6), Art. 17 Abs. 3 VO Nr, 38/64/EWG (Fn. 6) sowie Art. 10 Abs. 3 VO (EWG) Nr. 1612/68 (Fn. 4); zu erfolglosen Versuchen der Kommission, dieses Kriterium zu streichen, s. S. Goedings, Labor Migration (Fn. 3), S. 164 f., 220-222, 245-248.

9 Mit der RL 2004/38/EG (Fn. 6) wurde das Kriterium aufgegeben; nichts anderes galt zuvor bereits für die Freizügigkeit von wirtschaftlich inaktiven Unionsbürgern (Studierende, Rentner, Sonstige) aufgrund RL 90/364/ EWG (ABl. EG 1990 L 180/26), RL 90/365/EWG(ABl. EG 1990 L 180/28) und RL 93/96/EWG (ABl. EG 1993 L $317 / 59$ ).

10 Vgl. RL 64/221/EWG (ABl. EG 1964, S. 850) sowie, heute, Art. 27 ff. RL 2004/38/EG (Fn. 6); für Einzelheiten s. K. Hailbronner, Asyl- und Ausländerrecht, 3. Aufl. 2013, S. 453 ff.

11 Ausreichende finanzielle Existenzmittel verlangt Art. 7 Abs. 1 lit. b RL 2004/38/EG (Fn. 6) einzig von wirtschaftlich inaktiven Unionsbürgern - nicht von Arbeitnehmern; letztere haben aufgrund Art. 7 VO (EU) Nr. 492/2011 (Fn. 4) regelmäßig Anspruch auf gleichen Zugang zu Sozialleistungen, deren Bezug dem Arbeitnehmerstatus zumeist nicht entgegensteht; vgl. EuGH, Rs. 139/85 (Kempf), Slg. 1986, 1741 und der gemäß EuGH, Rs. 157/84 (Frascogna), Slg. 1985, 1739 auch für Familienmitglieder gilt.

12 Speziell eine mögliche Arbeitslosigkeit wurde während der Verhandlungen nicht diskutiert, weil man von Vollbeschäftigung und einer schnell verfügbaren Anschlussbeschäftigung ausging, vgl.S. Goedings, Labor Migration (Fn. 3), S. 259-264. 
weise neues Phänomen, das seinerzeit nicht auf der auf der politischen Agenda stand. ${ }^{13}$

Dementsprechend darf von Unionsbürgern nicht verlangt werden, dass sie die Landessprache erlernen - und zwar von Arbeitnehmern ebenso wenig wie von wirtschaftlich inaktiven Unionsbürgern im Anwendungsbereich des allgemeinen Freizügigkeitsrechts. ${ }^{14}$ Ein Portugiese darf sein ganzes Leben in Marseille verbringen, ohne ein Wort französisch zu erlernen - und ein Deutscher, der nach Wien zieht, hat einen Anspruch auf Familiennachzug mit seiner Ehefrau aus einem beliebigen Drittstaat, ohne dass diese Deutsch oder irgendeine andere Amtssprache der EU verstehen muss. ${ }^{15}$ Über die primärrechtliche Zulässigkeit einer hypothetischen Änderung der Richtlinie 2004/38/EG, der von Unionsbürgern und/oder der Familie jedenfalls in bestimmten Konstellationen das Erlernen der jeweiligen Landessprache des Aufenthaltsstaats einforderte, mag man sinnieren. ${ }^{16}$ Politisch jedenfalls sind derartige Änderungen illusorisch; ${ }^{17}$ de facto ist die Sekundärrechtslage änderungsfest. ${ }^{18}$

Damit verfolgt der EU-Gesetzgeber in der Sache einen vergleichsweise simplen Ansatz, den der EuGH mehrfach aufgriff: die effektive Verwirklichung der Freizügigkeit verlangt nach der Vorstellung der EU-Organe eine möglichst umfassende Familienzusammenführung, ${ }^{19}$ auch weil dies nach Meinung des EU-Gesetzgebers „Hindernisse beseitigt“ und ein gesicherter Aufenthaltsstatus „die Integration der Familie im Aufnahmeland" fördert. ${ }^{20}$ Auf empirische Erkenntnisse stützt sich diese Annahme nicht. ${ }^{21}$ Dessen ungeachtet müssen entgegenstehende öffentliche Belange

13 Vgl. C. Joppke, Citizenship and Immigration, 2010, Kap. 4.

14 Dies folgt schlicht aus dem Umstand, dass Art. 7 ff. RL 2004/38/EG (Fn. 6) diese Bedingung für keine der genannten Personengruppen aufführt und somit die Mitgliedstaaten diese innerstaatlich nicht einführen können; vgl. auch C. Costello, Metock: Free Movement and 'Normal Family Life' in the Union, CMLRev. 2009, S. 587 (602 f.).

15 Sowohl Art. 2 Nr. 2 RL 2004/38/EG (Fn. 6) als auch die EuGH-Rechtsprechung erstrecken den Familiennachzug auf Familienmitglieder aus Drittstaaten.

16 Wenn aufgrund einer hypothetischen Änderung der RL 2004/38/EG (Fn. 6) der Aufenthalt aller oder bestimmter Personengruppen an einfache Sprachkenntnisse geknüpft würde, begründete dies eine sekundärrechtliche Beschränkung des Art. 45 AEUV bzw. des Art. 21 AEUV durch den EU-Gesetzgeber, deren primärrechtliche Rechtfertigung der EuGH wohl zurückweisen würde; allenfalls könnte man sich vorstellen, dass in bestimmten Sonderkonstellationen - etwa bei langjähriger Arbeitslosigkeit - gewisse Integrationsanstrengungen, wie das Erlernen der Landessprache, nach Maßgabe einer strengen und am Einzelfall ausgerichteten Verhältnismäßigkeitsprüfung vom EU-Gesetzgeber gefordert werden könnten.

17 Eine Änderung verlangt einen Kommissionsvorschlag, der aus integrationspolitischen Gründen nicht ergehen dürfte, weil die EU-Organe die Freizügigkeit, durchaus zu Recht, der EU-Verfassungsidentität zuordnen; ebenso wie die Kommission frühere Versuche zur Änderung der RL 2004/38/EG (Fn. 6), etwa nach dem Metock-Urteil, abblockte.

18 Zur faktisch erschwerten Rechtsänderung als Machtgewinn für den EuGH siehe A. Stone Sweet, The European Court of Justice, in: Craig/de Búrca (Hrsg.), The Evolution of EU Law, 2. Aufl. 2011, S. 121 (126 ff.); der EuGH setzt bei unklaren Formulierungen im Zweifel auf Kontinuität und weist Änderungen zurück, vgl. D. Thym, Hindernisse (Fn. 1), S. 510 f.

19 Anschaulich EuGH, Rs. C-413/99 (Baumbast und R.), Slg. 2002, 1-7091, Rn. 50 sowie EuGH, Rs. C-370/90 (Singh), Slg. 1992, I-4265, Rn. 19 und EuGH, Rs. C-291/05 (Eind), Slg. 2007, I-10719, Rn. 22.

20 Zitate nach Erwgr. 5 VO (EWG) Nr. 1612/68 (Fn. 4) sowie dem heutigen Erwgr. 6 VO (EU) Nr. 492/2011 (Fn. 4).

21 Italienische Wanderarbeitnehmer haben grundsätzlich mit ähnlichen Integrationsproblemen in Deutschland zu kämpfen wie russische Neuzuwanderer; speziell der Bildungserfolg italienischer Schüler bleibt unbefriedigend; vgl. den 8. Bericht der Beauftragten über die Lage der Ausländerinnen und Ausländer in Deutschland, Juni 2010, S. $93 \mathrm{f}$. 
- wie die Förderung von Sprachkenntnissen - angesichts der klaren Entscheidung des Gesetzgebers hinter der Familieneinheit zurückstehen. Die Unionsbürgerschaft fungiert in diesen Fällen gleichsam als Migrationssteuerungsabwehranspruch gegenüber nationalstaatlichen Steuerungsanliegen. ${ }^{22}$ Insoweit verfolgen die EuGHRechtsprechung sowie die EU-Gesetzgebung eine klare inhaltliche Ausrichtung, die die Familieneinheit gleichsam als menschenrechtlichen Selbstzweck anerkennt und nahezu umfassend und ohne Ausnahme schützt. ${ }^{23}$

\section{Rechtsprechung zu Familienmitgliedern}

Dogmatisch entspringt die großzügige Regelung des Familiennachzugs keinem vertragsunmittelbaren Recht, das - ähnlich Art. 8 EMRK bzw. Art. 7 GRC - die Familie um ihrer selbst Willen schützte. Stattdessen besitzt der Familiennachzug nach der Konzeption des EuGH einen dienenden Charakter, weil ohne Einreise und Aufenthalt von Ehepartnern, Kindern und Eltern ,dem Aufenthaltsrecht (der Unionsbürger) jede praktische Wirksamkeit genommen (würde)." ${ }^{24}$ Aus diesem dienenden Charakter des abgeleiteten Rechts auf Familiennachzug folgt, dass der EuGH die Nachzugsrechte in unterschiedlichen Konstellationen aus den Unionsbürgerrechten ableitete (1.). Eben diese Entwicklung erreichte mit dem Ruiz Zambrano-Urteil einen kritischen Punkt, weil das Urteil den transnationalen Charakter des EU-Binnenmarkts mit seiner Orientierung an der grenzüberschreitenden Freizügigkeit zu überwinden schien (2.). Obwohl der EuGH in späteren Entscheidungen zurückwich und den Paradigmenwechsel ausbremste (3.), bereitet die Umsetzung des Urteils den innerstaatlichen Gerichten bis heute einige Probleme, die abschlieBend in den Blick genommen werden (4.).

\section{Beständige Ausweitung des Freizügigkeitsrechts}

Sekundärrecht schützte den Familiennachzug seit den 1960er-Jahren einzig bei der Übersiedlung in andere Mitgliedstaaten. ${ }^{25}$ Dennoch stellte der Gerichtshof im Singh-Urteil fest, dass die Arbeitnehmerfreizügigkeit ein Rückkehrrecht in den Herkunftsstaat mit dem Ehepartner umfasst. ${ }^{26}$ Gemäß den Entscheidungen in den Rechtssachen Jia und Eind gilt dies auch dann, wenn ein Unionsbürger den Ehepartner mit der Staatsangehörigkeit eines Drittstaats erst im Zielstaat kennenlernte

22 So $J$. Bast, Aufenthaltsrecht und Migrationssteuenung, 2011, S. 52 ff, sowie D. Thym, Freizügigkeit in Europa als Modell?, EuR 2011, S. $487(488-490)$

23 I.d.S. etwa $E$. Spaventa, From Gebhard to Carpenter: towards a (non-)economic European constitution, CMLRev. 2004, S. 743; A. Epiney, Von Akrich über Jia bis Metock, EuR 2008, S. 840 (847-853); A. Tryfonidou, Family Reunification Rights of (Migrant) Union Citizens, ELJ 2009, S. 634.

24 EuGH, Rs. C-200/02 (Zhu und Chen), Slg. 2004, I-9925, Rn. 45; erstmals zum Charakter als abgeleitete Rechte EuGH, Rs. 40/76 (Kermaschek), Slg. 1976, 1669, Rn. 7.

25 Vgl. Art. 10-12 VO (EWG) Nr. 1612/68 (Fn. 4)

26 EuGH, Rs. C-370/90 (Singh), Slg. 1992, I-4265, Rn. 19-21; näher S. Alber, Das Recht auf Schutz des Familienlebens im Aufenthaltsrecht, in: Bröhmer et al. (Hrsg.), Internationale Gemeinschaft und Menschenrechte. FS Ress, 2005, S. 371 (376 ff.). 
und sodann mit diesem in den Heimatstaat zurückkehrt. ${ }^{27}$ Schließlich kann nach dem Urteil Carpenter sogar der Familiennachzug im Heimatstaat dem Unionsrecht unterfallen, wenn ein Unionsbürger grenzüberschreitende Dienstleistungen in anderen Mitgliedstaaten erbringt. ${ }^{28}$ In Deutschland führte dies zwischenzeitlich dazu, dass findige Anwälte eine Eheschließung in Dänemark anrieten - um sodann als rückkehrender Dienstleistungsempfänger den Familiennachzug in Deutschland zu sichern. ${ }^{29}$ Dem schob das BVerwG freilich einen Riegel vor, weil von der Freizügigkeit durch die passive Dienstleistungsfreiheit nicht hinreichend effektiv Gebrauch gemacht worden sei. ${ }^{30}$ Sobald der Grenzübertritt jedoch eine deutlich gröBere Intensität besitzt als eine Eheschließung nebst Hochzeitsreise, greifen die Privilegien. ${ }^{31}$

Es überrascht kaum, dass der EuGH die großzügige Rechtsprechung für Arbeitnehmer und Selbständige auf das neu geschaffene Freizügigkeitsrecht der wirtschaftlich inaktiven Unionsbürger nach Art. 21 AEUV übertrug. Gemäß den Entscheidungen Baumbast ${ }^{32}$ sowie Zhu und Chen ${ }^{33}$ können Eltern aus Drittstaaten ein abgeleitetes Aufenthaltsrecht beanspruchen, wenn sie gemeinsam mit Kleinkindern, die die Staatsangehörigkeit eines Mitgliedstaats besitzen, im europäischen Ausland leben $^{34}$ (auch wenn in derartigen Konstellationen der dienende Charakter des elterlichen Aufenthaltsrechts deutlich wird, weil dieses regelmäßig endet, wenn das Kind erwachsen wird und der elterlichen Sorge nicht mehr bedarf ${ }^{35}$ ). Hierbei beeindruckt

27 S. EuGH, Rs. C-1/05 (Jia), Slg. 2007, I-1 und EuGH, Rs. C-291/05 (Eind), Slg. 2007, I-10719; ausführlicher G. Barrett, Family Matters: European Community Law and Third-Country Family Members, CMLRev. 2003, S. 369 ( 377 ff.).

28 S. EuGH, Rs. C-60/00 (Carpenter), Slg. 2002, I-6279 mit einer krit. Anm. von U. Mager, Dienstleistungsfreiheit und Schutz des Familienlebens, JZ 2003, S. 204; dies gilt freilich nur für die Personenfreiheiten (Art. 45, 49, 56 AEUV) und nicht für die Warenverkehrsfreiheit; zu letzterem wegen des fehlenden personalen Substrats im Ergebnis zutreffend VGH München, Beschluss v. $9.5 .2012,10$ CS 12.243 \& 244.

$29 \mathrm{Vgl}$. http://www.xn--hochzeit-dnemark-4nb.de/de/ [letzter Zugriff am 25.11.2013]: „eine modeme, absolut legale und kostengünstigste Möglichkeit,... mit (der) Sie Ihren Residenzstatus in Deutschland und in der Europäischen Union sichern können.".

30 S. BVerwGE 138, 353; restriktiv zu den aufenthaltsrechtlichen Folgen der passiven Dienstleistungsfreiheit auch EuGH, Rs. C-200/02 (Zhu und Chen), Slg. 2004, I-9925, Rn. 22 f.

31 Es liegt $m$.E. nahe, dass die passive Dienstleistungsfreiheit regelmäßig nicht die notwendige Intensität vermittelt; erforderlich ist vielmehr eine dauerhafte aktive Wirtschaftstätigkeit (wie in den Fällen Carpenter oder Singh) bzw. ein Aufenthalt von wirtschaftlich Inaktiven i.R.d. Art. 21 AEUV für mehrere Monate; in diesem Sinn zwischenzeitlich EuGH, Urteil v. 12.3.2014, Rs. C-456/12 (O. und B.); sowie EuGH, Urteil v. 12.3.2014 Rs. C-457/12 (S. und G.)

32 EuGH, Rs. C-413/99 (Baumbast und R.), Slg. 2002, I-7091, Rn. 50 ff. stützt sich auf das sekundärrechtliche Aufenthaltsrecht der Kinder nach Art. 12 VO (EWG) Nr. 1612/68 (Fn. 4); näher N. Reich, Citizenship and family on trial: a fairly optimistic overview of recent court practice with regard to free movement of persons, CMLRev. 2003, S. 615 (632f.).

33 EuGH, Rs. C-200/02 (Zhu und Chen), Slg. 2004, I-9925, Rn. 24 ff. stützt sich auf Art. 21 AEUV bzw. RL 2004/38/EG (Fn. 6).

34 Dies wurde nach Erlass der RL 2004/38/EG (Fn. 6) mit Blick auf Art. 10 VO(EU) Nr. 492/2011 (Fn. 4) bekräftigt von EuGH, Rs. C-310/08 (Ibrahim), Slg. 2010, I-1065 und Rs. C-480/08 (Teixeira), Slg. 2010, I-1107; näher $P$. Starup/M. Elsmore, Taking a Logical or Giant Step Forward? Comment on Ibrahim and Teixeira, ELRev. 2010, S. 571 .

35 S. EuGH, Rs. C-480/08 (Teixeira), Slg. 2010, I-1107, Rn. 76-87; in den meisten Mitgliedstaaten werden Eltern nach langjähriger Sorge ein eigenständiges Aufenthaltsrecht kraft nationalen Rechts erlangt haben; deutlich wird der dienende Charakter auch, wenn Familienmitglieder nach EuGH, Rs. C-10/05 (Mattern und Cikotic), Slg. 2006, I-3145, Rn. 25 keine EU-Binnenfreizügigkeit in Form der Arbeit in anderen Mitgliedstaaten als dem Aufenthaltsstaat besitzen. 
es den EuGH nicht, dass in einigen Fällen der Grenzübertritt in erster Linie von der Motivation getragen wurde, kraft Unionsrechts einen gesicherten Aufenthaltsstatus für die Familie zu erlangen. Seit dem Akrich-Urteil steht fest, dass die Ausübung der Freizügigkeit mit dem Ziel eines gesicherten Aufenthaltsstatus keinen Missbrauch darstellt. ${ }^{36}$ Einzig Scheinehen oder sonstige Betrugstatbestände können als Missbrauch geahndet werden ${ }^{37}$

Eine weitere Verselbständigung des Familiennachzugs folgte bald. Ein Verstoß gegen Unionsrecht soll nach dem Metock-Urteil auch dann vorliegen, wenn kein Mitgliedstaat dem Ehepartner zuvor die Einreise genehmigte - obgleich der Gerichtshof in Akrich wenige Jahre zuvor noch anderer Meinung gewesen war. ${ }^{38}$ Dies gilt selbst dann, wenn ein Drittstaatsangehöriger illegal eingereist war. Dass in derartigen Fällen nach herkömmlicher Dogmatik der Grundfreiheiten eine Beschränkung des Freizügigkeitsrechts des Unionsbürgers, zu dem der Nachzug erfolgt, nur schwer begründbar ist, weil der Unionsbürger infolge des Grenzübertritts nicht schlechter behandelt wird, als wenn er die innereuropäischen Grenzen nicht überschritten hätte, änderte das Ergebnis des EuGH nicht. ${ }^{39}$ Luxemburg legte die Richtlinie 2004/38/ EG in Metock im Lichte des Primärrechts so aus, dass auch in diesen Fällen ein Recht auf Familiennachzug besteht. ${ }^{40}$ Damit schritt ein Paradigmenwechsel voran, der in der Gesetzgebung und der Rechtsprechung von Anfang an angelegt war-und mit den genannten Urteilen in den Vordergrund tritt: Der EuGH schützt die Familieneinheit zunehmend als Selbstzweck, wenn auch dogmatisch konstruiert als Beschränkung der Freizügigkeit der Unionsbürger.

\section{Aufruf zur Revolution: Ruiz Zambrano}

Mit dem Schutz des Familienlebens als Selbstzweck verabschiedete sich der EuGH Schritt für Schritt von der funktionalen Grundierung einer ,Marktbürgerschaft, “ die sich auf die transnationale Förderung individueller Freiheit konzentriert. Es erschien, auf den ersten Blick jedenfalls, eine Bürgerschaft jenseits des Marktes zu entstehen, die freilich dogmatisch als Beschränkung der Unionsbürgerfreizügigkeit konzipiert war und insofern in der etablierten Grundstruktur des Binnenmarkts gründete. ${ }^{41}$ Dies änderte sich im März 2011, als der EuGH in Ruiz Zambrano eine konzeptionelle Schwelle überschritt, die einzelne Kommentatoren als Rubikon auf

36 EuGH, Rs. C-109/01 (Akrich), Slg. 2003, I-9607, Rn. 55 f.; ausfuihrlicher C. Costello, Citizenship of the Union: Above Abuse?, in: de la Feria/Vogenauer (Hrsg.), Prohibition of Abuse of Law, 2011, S. 321.

37 Vgl. Art. 35 RL 2004/38/EG (Fn. 6) sowie EuGH, Rs. C-109/01 (Akrich), Slg. 2003, I-9607, Rn. 57.

38 S. EuGH, Rs. C-127/08 (Metock), Slg. 2008, 1-6241; ausführlicher S. Currie, Accelerated Justice or a Step too Far?, ELRev. 2009, S. 310.

39 Kritisch A. Epiney, Akrich (Fn. 23), S. 847 f. und N. Nic Shuibhne, The Outer Limits of EU Citizenship: Displacing Economic Free Movement Rights?, in: Barnard/Odudu (Hrsg.), The Outer Limits of European Union Law, 2009, S. 167 (179 f.).

40 EuGH, Rs. C-127/08 (Metock), Slg. 2008, 1-6241, Rn. 49 ff.

41 S. N. Nic Shuibhne, The Resilience of EU Market Citizenship, CMLRev. 2010, S. 1597 (1611-1618) und F. Wollenschläger. Die Unionsbürgerschaft und ihre Dynamik für den Integrationsprozess jenseits des Marktes, ZEuS 2009, S. 1 (45-48). 
dem Weg zur „wahrhaftigen“ Bürgerschaft einstuften. ${ }^{42}$ Bereits der Sachverhalt zeugte vom fehlenden Binnenmarktbezug: Herr Ruiz Zambrano war kolumbianischer Staatsangehöriger, der sich nach der Ablehnung seines Asylantrags ohne Genehmigung in Belgien aufhielt. Verschiedene Legalisierungsversuche scheiterten und der Bezug zur Unionsbürgerschaft ergab sich erst, als zwei Kinder geboren wurden, die als Staatenlose unmittelbar die belgische Staatsangehörigkeit erlangten und damit Unionsbürger wurden..$^{43}$

Den Schritt des EuGH weg von der transnationalen Marktbürgerschaft verdeutlichen eine dogmatische und eine theoretische Überlegung.

Erstens das dogmatische Argument. Der EuGH hätte das Urteil bei einer großzügigen Betrachtung auf Grundlage der etablierten Rechtsprechung durch eine (sehr) weite Ausdehnung des Beschränkungsbegriffs lösen können, unter Verweis auf die Beeinträchtigung des potentiellen, zukünftigen Freizügigkeitsrechts der Kinder ${ }^{44}$ - oder in Übereinstimmung mit den Mitgliedstaaten sowie der Kommission die Sache dem nationalen Recht sowie dem EGMR überantworten können. ${ }^{45}$ Die Große Kammer entschied sich jedoch gegen diesen Weg und stellte stattdessen kurz und knapp fest, dass zwar kein grenzüberschreitender Bezug vorliege und auch die Freizügigkeits-Richtlinie 2004/38/EG nicht gelte. ${ }^{46}$ Dies stehe der Berufung auf die Unionsbürgerschaft jedoch nicht entgegen. Nach Meinung des EuGH sind nationale Maßnahmen unzulässig, die ,den tatsächlichen Genuss des Kernbestands der Unionsbürgerrechte verwehren." 47

Innerhalb des postulierten „Kernbereichs“ ist kein grenzüberschreitender Bezug erforderlich. ${ }^{48}$ Wichtig ist das Bewusstsein für die Eigenständigkeit des Kernbereichsarguments. Dieses folgt direkt aus dem Wesen der Unionsbürgerschaft bzw. Art. 20 AEUV und ist damit unabhängig von speziellen Freizügigkeitsgewährleistungen..$^{49}$ Oder anders formuliert: Es gibt Situationen, in denen aus der Unionsbürgerschaft rechtliche Schranken für die Mitgliedstaaten folgen, die von den speziellen Rechtsgewährleistungen des Primär- und Sekundärrechts nicht umfasst sind.

Zweitens folgt hieraus in theoretischer Hinsicht: Mit der Erstreckung der Unionsbürgerschaft auf reine Inlandssachverhalte in Bezug auf arbeitslose und drittstaatsangehörige Eltern von Unionsbürgern lässt der EuGH die transnationale Fundierung

42 So M. Nettesheim, Der „Kernbereich“ der Unionsbürgerschaft, JZ 2011, S. 1030 (1032 f.) und D. Kochenov, A Real European Citizenship: A New Jurisdiction Test: A Novel Chapter in the Development of the Union in Europe, CJEL 2011, S. 55 ( 95 ff.).

43 Zu den nachfolgenden Überlegungen bereits D. Thym, Hindernisse (Fn. 1), S. $166 \mathrm{f}$

44 Vgl. M. Wendel, in: Grabenwarter (Hrsg.), Enzyklopädie Europarecht, Bd, 2, 2014, \& 22, Rn. 115; A. Lansbergen/ N. Miller, European Citizenship Rights in Internal Situations, EuConst 2011, S. 287 (294-296) und D. Kochenov, Real European Citizenship (Fn. 42), S. 74-91.

45 Vgl. EuGH, Rs. C-34/09 (Ruiz Zambrano), Slg. 2011, I-1177, Rn. 37.

46 S. EuGH, Rs. C-34/09 (Ruiz Zambrano), Slg. 2011, I-1177, Rn. 39

47 EuGH, Rs. C-34/09 (Ruiz Zambrano), Slg. 2011, I-1177, Rn. 42.

48 Ausführlicher $K$. Hailbronner/D. Thym, Ruiz Zambrano - Die Entdeckung des Kernbereichs der Unionsbürgerschaft, NJW 2011, S. 2008 (2009) und A. Wiesbrock, Disentangling the „Union Citizenship Puzzle”? The McCarthy Case, ELRev. 2011, S. 861 ( 865 ff.).

49 Näher K. Hailbronner/D. Thym, Ruiz Zambrano (Fn. 48), S. 2009 f. sowie P. Van Elsuwege/D. Kochenov, On The Limits of Judicial Intervention: EU Citizenship and Family Reunification Rights, EJML 2011, S. 443 (450-452). 
der Marktbürgerschaft hinter sich. Es geht weder um den Binnenmarkt noch um transnationale Rechte, weil die jungen Unionsbürger nie in einem anderen Mitgliedstaat als ihrem Heimatstaat lebten. Damit betrifft das Urteil im Kern den Schutz des Menschen um seiner selbst Willen und mithin eine Frage der sozialen Gerechtigkeit. Eben dies meinen Kommentatoren, wenn sie den EuGH loben, weil er mit der Abkehr von der Marktbürgerschaft den Rubikon zur ,wahrhaftigen“ Bürgerschaft überschritten habe. ${ }^{50}$ An die Stelle der Effektuierung der grenzüberschreitenden Freizügigkeit tritt der Schutz des Menschen um seiner selbst Willen. Der EuGH kehrt der Marktbürgerschaft den Rücken und wendet sich Fragen der sozialen Gerechtigkeit und des Aufenthaltsrechts zu, für die die Grundfreiheiten nicht direkt geschaffen wurden.

Es dürfte kein Zufall sein, dass der EuGH sein Auslegungsergebnis in der überaus knappen Begründung des Ruiz Zambrano-Urteils auf den „Geist“51 der Verträge stützte - eine Teleologie, die der Gerichtshof durch die beschwörende Wiederholung der Formel vom, ,grundlegenden Status“52 unterstreicht (zusätzlich könnte man allenfalls noch auf eine subtile Änderung im Vertragstext verweisen, die der EuGH jedoch nicht anführt ${ }^{53}$ ). An die Stelle des geschriebenen Primär- und Sekundärrechts tritt eine - zumeist vage - Vorstellung vom Endziel einer „echten“ Bürgerschaft. Der Rückzieher des Gerichtshofs in Folgeverfahren dürfte auch daran liegen, dass die abstrakte Beschwörung der Bürgerschaft nicht ausreicht, weil verschiedene Personen je nach Standpunkt unter einer echten supranationalen Bürgerschaft mit guten Gründen unterschiedliche Dinge verstehen. ${ }^{54}$

\section{Ausgebremster Paradigmenwechsel}

In der Rückschau war das Ruiz Zambrano-Urteil weniger revolutionär als es auf den ersten Blick schien, weil zum Zeitpunkt der Urteilsverkündung keineswegs feststand, dass der Gerichtshof in Folgeentscheidungen eine restriktive Linie einschlagen würde, die die konzeptionellen Folgewirkungen einfängt. Der EuGH mag sich in Ruiz Zambrano für einen großen Sprung nach vorn positioniert haben, musste aus strukturellen Gründen jedoch auf halbem Weg stehen bleiben. Aus den Folgeurteilen ergibt sich in dogmatischer Hinsicht, dass der Kernbereichsschutz restriktiv zu handhaben ist. Zudem wird in theoretischer Hinsicht die transnationale

50 Wiederum M. Nettesheim, „Kernbereich“ (Fn. 42), S. 1032 f. und D. Kochenov, Real European Citizenship (Fn. 42), S. $95 \mathrm{ff}$.

51 EuGH, Rs. 26/62 (van Gend en Loos), Slg. 1963, 3 (27) und Rs. $6 / 64$ (Costa/E.N.E.L.), Slg. 1964, 1251 (1269).

52 Erneut EuGH, Rs. C-34/09 (Ruiz Zambrano), Slg. 2011, I-1177, Rn. 42.

53 Zur Änderung des Art. 20 AEUV von der (nachgelagerten) „Ergänzung“ hin zum (gleichrangigen) „Hinzutreten", was vom EuGH nicht aufgegriffen wird, s. A. Schrauwen, European Citizenship in the Treaty of Lisbon, Maastricht J. Eur. \& Comp. L. 2008, S. 55 (59-60); H. de Waele, The Ever-Evolving Concept of EU Citizenship, in: Talani (Hrsg.), Globalisation, Migration and the Future of Europe, 2011, S. 191 (192-194); D. Kochenov/R. Plender, EU Citizenship: From an Incipient Form to an Incipient Substance? The Discovery of the Treaty Text, ELRev. 2012, S. 369 (384-388).

54 Zur fehlenden Inhaltsschärfe des Teleologie-Arguments wegen der Offenheit des Bürgerschaftskonzepts siehe D. Thym, Towards 'Real' Citizenship? The Judicial Construction of Union Citizenship and its Limits, in: Adams et al. (Fn. 1), S. $155(161-163)$ 
Grundierung der Unionsbürgerrechte reaktiviert. Damit ist der Paradigmenwechsel hin zur ,wahrhaften" Bürgerschaft ausgebremst.

Die zentrale Einschränkung vollzog der Gerichtshof in den Urteilen McCarthy und Dereci. ${ }^{55}$ In diesen beiden Entscheidungen rekonstruiert der Gerichtshof den Kernbereichsschutz als ein Kriterium, das sich ,auf Sachverhalte bezieht, die dadurch gekennzeichnet sind, dass sich der Unionsbürger de facto gezwungen sieht, nicht nur das Gebiet des Mitgliedstaats, dem er angehört, zu verlassen, sondern das Gebiet der Union als Ganzes. " ${ }^{56}$ Damit steht fest, dass das Kernbereichsargument vorrangig Sonderfälle betrifft, ${ }^{57}$ wenn mit dem Verlust der nationalen Staatsangehörigkeit die Existenz des Unionsbürgerstatus auf dem Spiel steht (Urteil Rottmann ${ }^{58}$ ) oder eine Pflicht zum Verlassen des Unionsgebiets droht (Urteil Ruiz Zambrano). Dogmatisch gesprochen ist Art. 20 AEUV kein Beschränkungsverbot (restriction), sondern verbietet nur den rechtlichen oder faktischen Entzug der Unionsbürgerrechte (deprivation). ${ }^{59}$ Es geht mithin um Sonderfälle eines "Seins oder Nichtseins“ der Unionsbürgerrechte. Jenseits dieser hohen Hürde gelten - nach der Regieanweisung im Dereci-Urteil - die Grundrechte. ${ }^{60}$

Zugleich stellt der Gerichtshof klar, dass die restriktive Ausrichtung des Kernbereichstests nicht durch eine großzügige Auslegung des Art. 21 AEUV umgangen werden kann, indem einzelne Unionsbürger ,rein hypothetische“ Beschränkungen des Freizügigkeitsrechts geltend machen. ${ }^{61}$ Damit hat der Gerichtshof die Kernbereichsdoktrin relativiert. Diese beschränkt sich für die Zwecke des Familiennachzugs auf Situationen, in denen ein Unionsbürger kraft nationalen Rechts rechtlich oder faktisch zum Verlassen des Unionsgebiets gezwungen wird. Dieses Kriterium ist nach Meinung des EuGH speziell bei Eltern von Kleinkindern erfüllt (s. das Urteil Ruiz Zambrano) - während die Abschiebung eines Ehegatten nach dem DereciUrteil regelmäßig nicht den Kernbestand beeinträchtigen soll, weil die bloße Tatsache, dass der Inlandsaufenthalt des Ehegatten ,aus wirtschaftlichen Gründen oder

55 Das McCarthy-Urteil war eine Entscheidung einer Kammer von füf Richtern unter Vorsitz des heutigen EuGHVizepräsidenten Koen Lenaerts; der (restriktiven) Einschätzung dieser Richter folgte die Große Kammer einige Monate später im Urteil Dereci.

56 EuGH, Rs. C-256/11 (Dereci u.a.), Slg. 2011, I-11315, Rn. 60; ebenso zuvor EuGH, Rs. C-434/09 (McCarthy), Slg. 2011, 1-3375, Rn. 50; zur Herkunft der Formel s. N. Nic Shuibhne, (Some Of) The Kids Are All Right, CMLRev. 2012, S. 349 (361 f., 367 f.) sowie, mittelbar, N. Graf Vitzthum, Die Entdeckung der Heimat der Unionsbürger, EuR 2011, S. 550.

57 Der EuGH selbst betont in allen Urteilen den Ausnahmecharakter eines Rückgriffs auf das Kernbereichsargument; vgl. M. Wendel, in: Grabenwarter (Fn. 44), § 22, Rn. 110.

58 Vgl. EuGH, Rs. C-135/08 (Rottmann), Slg. 2010, I-1449, Rn. 42 f. sowie D. Kochenov, Real European Citizenship (Fn. 42), S. 75-80 und F. Schoch, Europäisierung des Staatsangehörigkeits- und Aufenthaltsrechts durch den „Unionsbürgerstatus“, in: Jochum et al. (Hrsg.), Grenzüberschreitendes Recht-Crossing frontiers. FS Hailbronner, 2013, S. 355-368. S. auch K. Traunmüller in diesem Heft auf S. 33.

59 Hierzu K. Lenaerts, The Court's Outer and Inner Selves. Exploring the External and Internal legitimacy of the European Court of Justice, in: Adams et al. (Fn. 1), S. 13 (50 f.); und S. Reynolds, Exploring the "Intrinsic Connection" between Free Movement and the Genuine Enjoyment Test, ELRev. 2013, S. 376 (385 f.).

60 Näher zu den Grundrechten unten IV.

61 So EuGH, Urteil v. 8.11.2012, Rs. C-40/11 (lida), Rn. 77; s. auch die Stimmen in Fn. 44, wonach der EuGH in Ruiz Zambrano von Anfang an eine großzügige Lesart des Beschränkungsverbots hätte annehmen können; diesen Weg schneidet der EuGH für die Zukunft ab; vgl. K. Lenaerts, Court's (Fn. 59), S. 53-55 und S. Reynolds, Exploring (Fn. 59), S. 386 f. 
zur Aufrechterhaltung der Familiengemeinschaft... wünschenswert erscheinen könnte... für sich genommen nicht die Annahme [rechtfertigt], dass der Unionsbürger [bei einer Verweigerung des Ehegattennachzugs] gezwungen wäre, das Gebiet der Union zu verlassen." 62

Dogmatisch überzeugend ist diese Konzentration der Kernbereichsdoktrin auf jugendliche Unionsbürger und deren drittstaatsangehörige Eltern nicht. Die allermeisten Leser würden sich emotional und damit faktisch sehr wohl zum Verlassen des Unionsgebiets gezwungen sehen, wenn der jeweilige Partner aus dem Unionsgebiet ausreisen müsste. ${ }^{63}$ Hinzu kommt, dass auch im Ruiz Zambrano-Urteil eine Ausreise der Kinder mit den Eltern faktisch nicht zu befürchten stand, weil ein belgisches Gericht die Abschiebung der Familie nach Kolumbien aus humanitären Gründen untersagt hatte ${ }^{64}$ und die Eltern zudem eine Arbeit im EU-Ausland unter Berufung auf die Urteile Metock sowie Zhu \& Chen hätten suchen können. ${ }^{65}$ Es ging in dem Rechtsstreit nicht um die tatsächliche Ausreise, sondern um die Verbesserung des Aufenthaltsstatus nebst Zugang zum Arbeitsmarkt. Diesen Wunsch erfüllte der EuGH in Ruiz Zambrano, nicht jedoch in Dereci.

Mit derartigen Gemeinsamkeiten und Unterschieden zwischen den behandelten Fällen befasst sich der EuGH jedoch nicht weiter. Die Richter scheinen froh zu sein, das Ruiz Zambrano-Problem mit der nachträglichen Rekonstruktion als Pflicht zum Verlassen des Unionsgebiets in den Griff bekommen zu haben und durch diese Lösung negativen Rückwirkungen auf die föderale Machtbalance zwischen der EU und den Mitgliedstaaten infolge einer expansiven Deutung der Kernbereichsdoktrin vorgebeugt zu haben. ${ }^{66}$ Binnen weniger Monate vollzog der Gerichtshof dasselbe Wechselspiel von Ausweitung und Einschränkung, welche die Rechtsprechung zum Warenverkehr mit den Entscheidungen Cassis de Dijon sowie Keck \& Mithouard vollzog. ${ }^{67}$ Die Kernbestandsdoktrin betrifft zum aktuellen Zeitpunkt zwei Konstellationen: Erstens den Erwerb und den Verlust der Unionsbürgerschaft aufgrund

62 EuGH, Rs. C-256/11 (Dereci u.a.), Slg. 2011, I-11315, Rn. 68; bekräftigt in EuGH, Urteil v. 8.5.2013, Rs. C-87/12 (Ymeraga und Ymeraga-Tafarshiku), Rn. 38 f., wenn der verweigerte Familiennachzug keine Verletzung des Kernbestands darstellt, weil keine speziellen Faktoren hinzukommen.

63 Ebenso A. Tryfonidou, Redefining the Outer Boundaries of EU Law, European Public Law 2012, S. 493 (515); polemisch zugespitzt der Ratschlag von $J .-Y$. Carlier, Purely internal situations and EU citizens' rights after the Zambrano, McCarthy, and Dereci judgments, Online Journal on Free Movement of Workers within the European Union No. 5 (2013), S. 8, dass Frau Dereci sich den Finger abschneiden sollte, um sodann mangels Arbeitsfähigkeit finanziell vom Ehemann abzuhängen, der in der Folge eventuell ein Aufenthaltsrecht bekäme, soweit infolge der Abhängigkeit eine Ausreise aus dem EU-Gebiet drohte.

64 Vgl. EuGH, Rs. C-34/09 (Ruiz Zambrano), Slg. 2011, I-1177, Rn. 15, auch wenn diese Entscheidung nach dem Ende des Bürgerkriegs dort heute eventuell keinen Bestand mehr hätte.

65 So zutreffend N. Nic Shuibhne, Kids (Fn. 56), S. 368

66 Ebenso D. Thym, Hindernisse (Fn. 1), S. 518-521; C. Calliess, The Dynamics of European Citizenship, in: CJEU (Hrsg.), The Court of Justice and the Construction of Europe, 2013, S. 425 (430-432); C. Langenfeld, Einwanderungssteuerung im Spannungsfeld zwischen nationalem und europäischem Recht, in: Breuer et al. (Hrsg.), Der Staat im Recht. Festschrift für Eckart Klein, 2013, S. 553 (561-566); und P. Van Elsuwege, European Union Citizenship and the Purely Internal Rule Revisited, EuConst 2011 , S. 308 (322 f).

67 So E. Sharpston, Citizenship and Fundamental Rights - Pandora's Box or a Natural Step towards Maturity?, in: Cardonnel et al. (Hrsg.), Constitutionalising the EU Judicial System. Essays in Honour of Pernilla Lindh, 2012. S. 245 (265 f.); sinngemäß D. Kostakopoulou, Co-Creating European Union Citizenship, C.Y.E.L.S. 2014, S. 255 (266 ff.) 
einer Ein- oder Ausbürgerung. Zweitens die faktische Ausreisepflicht von jugendlichen Unionsbürgern, wenn diese auf die Pflege und Sorge durch einen Elternteil aus einem Drittstaat angewiesen sind. ${ }^{68}$

Ungeachtet der nachfolgend zu diskutierenden Schwierigkeiten bei der Anwendung dieser Kriterien bleibt ein konzeptionelles Fazit. Mit der Formel von der faktischen Ausreisepflicht in der Folgerechtsprechung kehrt in abgeschwächter Form die funktionale Grundierung der Marktbürgerschaft zurück. Zwar ist weiterhin kein grenzüberschreitendes Element erforderlich, ${ }^{69}$ aber dennoch werden drittstaatsangehörige Familienmitglieder nicht direkt geschützt. In den jüngeren Urteilen akzentuiert der EuGH den dienenden Charakter des Familiennachzugs; die Begünstigten profitieren von ,abgeleitete[n] Rechte[n]..., die sie als Familienangehörige des Berechtigten erworben haben. "70 Geschützt werden pflegende Eltern und nicht Drittstaatsangehörige kraft ihres Menschseins. Gleiches gilt für Unionsbürger, deren Schutz der praktischen Wirksamkeit des Unionsrechts dient. Dies ist ein vertrautes Muster. Der Ausbau der Individualrechte fördert den Effet utile der Unionsrechtsordnung. ${ }^{71}$ Wenn die Unionsbürgerschaft den Schritt zum Schutz des Menschen um seiner selbst Willen nicht geht, muss dies kein Fehler sein. Dieser Schutz bleibt einer anderen Kategorie überantwortet: den Menschenrechten, auf die im letzten Abschnitt näher einzugehen sein wird.

\section{Probleme der innerstaatlichen Anwendung}

Verfassungsrechtlich sind die Grenzen der Kernbereichsdoktrin geklärt, seit der EuGH deren Folgewirkungen durch die nachträgliche Beschränkung auf die faktische Pflicht zum Verlassen des Unionsgebiets einfing. Damit ist jedoch nicht gesagt, dass nationale Gerichte hinreichende Klarheit besäßen, unter welchen Voraussetzungen eine Verletzung des Kernbereichs anzunehmen wäre. Leider besteht diese Rechtssicherheit nicht. Im Freizügigkeitsrecht verfehlt der Gerichtshof wieder einmal die Entwicklung klarer Leitlinien, die eine gleichförmige und zuverlässige Anwendung des Unionsrechts durch die nationalen Gerichte sicherstellen. ${ }^{72}$ Belastbare Rechtsmaßstäbe i.S. eines auf Präjudizien basierten, die Unterschiede zwischen Fallkonstellationen herausarbeitenden Fallrechts (case law) sind für die Kernbe-

68 Ungeachtet dieser eingeschränkten, rechtspraktischen Bedeutung besitzt die Kembereichsdoktrin eine hinreichende Abstraktion, um vom EuGH bei Bedarf reaktiviert und auf weitere Sachverhalte erstreckt zu werden.

69 Dogmatisch hält der EuGH an der Nichtgeltung der Unionsbürgerschaft in reinen Inlandssachverhalten fest, konzipiert die Kernbereichsdoktrin sodann jedoch als Frage, die wegen der Rückwirkungen auf die Unionsbürgerrechte gerade keinen rein internen Sachverhalt darstellt; vgl. hierzu EuGH, Rs. C-256/11 (Dereci u.a.), Slg. 2011, I-11315, Rn. 61 sowie $M$. Wendel, Aufenthalt als Mittel zum Zweck, DÖV 2014, S. 133 (142f.) und Reynolds, Exploring (Fn. 59), S. $377 \mathrm{f}$

70 Überraschend deutlich EuGH, Rs. C-256/11 (Dereci u.a.), Slg. 2011, 1-11315, Rn. 55 sowie Rn. 67 mit einem Verweis auf die praktische Wirksamkeit der Unionsbürgerschaft; ebenso EuGH, Urteil v. 8.11.2012, Rs. C-40/11 (Iida), Rn. 63, 67 f;; Urteil v. 6.12.2012, verb. Rs. C-356/11 u. C-357/11 (O. und S.), Rn. 56; Urteil v. 8.5.2013, Rs. C-87/12 (Ymeraga und Ymeraga-Tafarshiku), Rn. 35.

71 Vgl. C. Calliess, Der Unionsbürger, EuR-Beiheft 1-2007, S. 7 (9 f.).

72 Zu Unklarheiten beim Zugang zu Sozialleistungen M. Dougan, Bubble (Fn. 1), S. 140-145 sowie beim Aufenthaltsrecht von nichterwerbstätigen Unionsbürgern i.R.d. Art. 7 Abs. 1 lit. b RL 2004/38/EG (Fn.6) s. R. Rebhahn in diesem Heft auf S. 95 . 
reichs-Rechtsprechung nicht erkennbar. ${ }^{73}$ Dies ändert jedoch nicht den Inhalt der EuGH-Entscheidungen, die in dezisionistischer Manier inhaltliche Vorgaben tätigen und sich nicht zu einem kohärenten Ganzen fügen. Dies ist speziell für die Migrationsverwaltung bedauerlich, weil diese auf die möglichst gleichförmige Bearbeitung großer Antragszahlen anhand klarer Kriterien angewiesen ist. ${ }^{74}$ Diese fehlen bislang.

Fest steht aufgrund der bisherigen Urteile nur, dass die Kernbereichsdoktrin speziell bei jugendlichen Unionsbürgern relevant wird, wenn diese auf die Pflege und Sorge durch einen Elternteil aus einem Drittstaat angewiesen sind. In der Praxis bereitet speziell das letzte Kriterium der Pflege und Sorge durch ein Elternteil erhebliche Probleme, zumal der EuGH in der Entscheidung $O \& S$ feststellte, dass diese Voraussetzung auch bei Patchwork-Familien erfüllt sein kann, wenn die Drittstaatsangehörigen, deren Ausreise droht, kein förmliches Sorgerecht für die jugendlichen Unionsbürger besitzen und/oder als Stiefeltern nicht die leiblichen Väter und Mütter sind. ${ }^{75}$ Konkret sollen die nationalen Gerichte aufgrund einer Gesamtbetrachtung die „rechtliche, finanzielle oder affektive Sorge“ durch die Eltern im Einzelfall beurteilen, ob der Kernbereichsschutz beeinträchtigt wurde ${ }^{76}$ (freilich ergänzt um den Hinweis, dass diese notorisch offenen Kriterien aller Voraussicht nach nicht erfüllt seien ${ }^{77}$ ). Hierbei bezieht der Gerichtshof das Kriterientrias der rechtlichen, finanziellen oder affektiven Sorge vorrangig auf Kinder, ${ }^{78}$ sodass der Ehegattennachzug auch in der Zukunft regelmäßig nicht anhand dieser Kriterien mit Blick auf den Einzelfall betrachtet werden muss und weiterhin nicht den Kernbestand berührt. ${ }^{79}$ Man kann diese kategoriale Unterscheidung zwischen Ehegatten und Kindern damit zu begründen suchen, dass Kinder nach Art. 24 GRC besonderen Schutz genießen und mangels rechtlicher und faktischer Entscheidungsfreiheit intensiver von den Eltern abhängen als Ehegatten vom Lebenspartner, der zumindest im ersten Zugriff, anders als die Eltern, frei gewählt wurde..$^{80}$

Welche Anordnung soll ein innerstaatliches Gericht treffen, wenn dieses im Einzelfall zu dem Schluss kommt, dass die Kernbereichsdoktrin durch die Abschiebung

73 Ebenso S. Griller in diesem Heft auf S. 7 und N. Nic Shuibhne, Kids (Fn. 56), S. 379.

$74 \mathrm{Vgl}$. R. Wahl, Dic Aufgabenabhängigkeit von Verwaltung und Verwaltungsrecht, in: Hoffmann-Riem et al. (Hrsg.), Reform des Allgemeinen Verwaltungsrechts - Grundfragen, 1993, S. 177 (203-206).

75 Vgl. EuGH, Urteil v. 6.12.2012, verb. Rs. C-356/11 u. C-357/11 (O. und S.), Rn. 50-58.

76 EuGH, Urteil v. 6.12.2012, verb. Rs. C-356/11 u. C-357/11 (O. und S.), Rn. 56 (Zitat) und Rn. 53 (Verweis auf die notwendige Entscheidung im Einzelfall); s. auch $K$. Lenaerts, Court's (Fn. 59), S. 57 f. sowie BVerwG, Urteil v. $30.7 .2013,1 \mathrm{C} 15.12$, Rn. $30 \mathrm{ff}$.

77 EuGH, Urteil v. 6.12.2012, verb. Rs. C-356/11 u. C-357/11 (O. und S.), Rn. 57.

$78 \mathrm{Vgl}$. EuGH, Urteil v. 6.12.2012, verb. Rs. C-356/11 u. C-357/11 (O. und S.), Rn. 56 und der dort zitierte GA Bot, Schlussanträge v. 27.12.2012, Rn. 44 beziehen sich auf Kinder und bekräftigen für Ehegatten das DereciUrteil; ebenso einige Monate später EuGH, Urteil v. 8.5.2013, Rs. C-87/12 (Ymeraga und Ymeraga-Tafarshiku), Rn. 38 f.; missverständlich M. Almhofer, Zum Aufenthaltsrecht Drittstaatsangehöriger zwei Jahre post Ruiz Zambrano, NVwZ 2013, S. 1134 (1135), die so verstanden werden kann, als ob das Kriterientrias den Kernbereich abstrakt definiere (nicht nur in Bezug auf Kinder).

79 Allenfalls in Sonderkonstellationen einer besonderen finanziellen oder rechtlichen Abhängigkeit vom Ehegatten, etwa bei geistiger Behinderung, die über das „NormalmaB“ der (nach dem Dereci-Urteil nicht schützenswerten) wirtschaftlichen und emotionalen Bindung an den Ehegatten hinausgeht, wird man mit GA Bot, Schlussanträge v. 27.12.2012 (Fn. 78), Rn. 45 eine Ausnahme zu Gunsten von Ehepartnern annehmen können.

80 Sinngemäß bereits K. Hailbronner/D. Thym, Ruiz Zambrano (Fn. 48), S. 2013. 
eines Elternteils verletzt würde? Diese Frage ist anhand eines etablierten Argumentationsmusters zu beantworten: Soweit der EU-Gesetzgeber einen Sachbereich nicht geregelt hat, fungiert die Unionsbürgerschaft, ebenso wie die Grundfreiheiten, als Korrektiv der zweiten Stufe. Es gilt das einschlägige nationale Recht, dessen Aussagen im Konfliktfall im Lichte der Kernbereichsdoktrin zu korrigieren sind. ${ }^{81}$ In Deutschland sind auf den Familiennachzug mithin $\S \S 27 \mathrm{ff}$. AufenthG anzuwenden. ${ }^{82} \mathrm{Da}$ diese nationalen Bestimmungen bereits ein ausgefeiltes Rechtsregime errichten, das zudem im Lichte der Grundrechte auszulegen ist, dürfte das Kernbereichsargument in der Rechtspraxis selten durchgreifen, weil die meisten Interessen der Migranten bereits anderweitig abgedeckt werden. ${ }^{83}$ Hinzu kommt, dass auch der Kernbereichsschutz nicht absolut gilt, weil entgegenstehende Gemeinwohlbelange zu berücksichtigen sind ${ }^{84}$ Aus all diesen Gründen besteht strukturell kein prinzipieller Unterschied zum Prüfprogramm der Grundrechte mit der Ausnahme, dass die Kernbereichs-Rechtsprechung nicht die Familieneinheit als Selbstzweck mit Gemeinwohlbelangen abwägt, sondern den Aufenthalt der betroffenen EU-Bürger im Unionsgebiet schützt.

Ein großes Defizit der EuGH-Rechtsprechung sind die fehlenden Aussagen zu den Rechtsfolgen. Fest steht einzig, dass die Freizügigkeits-Richtlinie 2004/38/EG keine Anwendung findet, weil diese einen Grenzübertritt innerhalb der EU durch einen Unionsbürger voraussetzt. ${ }^{85}$ Gleiches gilt in Deutschland für das FreizügigG/ EU. ${ }^{86}$ Darüber hinaus bleibt bei der Umsetzung der EuGH-Urteile freilich unklar, ob dem Unionsrecht inhaltliche Vorgaben zur Qualität des Aufenthaltstitels zu entnehmen sind, der im Fall einer Verletzung der Kernbereichsdoktrin zu verleihen ist. Den Vorgaben des EGMR zu Art. 8 EMRK genügt regelmäßig eine Duldung, ${ }^{87}$ ohne dass Vorgaben zum Arbeitsmarktzugang oder sonstigen Begleitrechten getätigt würden. Eben dies könnte beim EuGH jedoch anders aussehen, weil das Ruiz Zambrano-Urteil auch den Arbeitsmarktzugang des Vaters betraf, ohne dass der EuGH hierzu eindeutige Aussagen tätigte..$^{88}$ Weitere Einzelheiten muss im Zweifelsfall der EuGH aufgrund einer erneuten Vorlage beantworten. In der Zwischenzeit gilt die allgemeine Regel: Nationale Gerichte haben das nationale Recht anzuwenden, soweit und solange die Kernbereichsdoktrin keine Vorgaben ausspricht, die abweichende Rechtsfolgen anordnen.

81 Vgl. K. Hailbronner/D. Thym, Ruiz Zambrano (Fn. 48), S. 2012 und K. Hailbronner, EU-Freizügigkeitsgesetz, Aufenthaltsgesetz und Assoziationsrecht, in: Bundesamt für Migration und Flüchtlinge (Hrsg.), Aufgaben und Herausforderungen. Ein Rückblick auf 60 Jahre Bundesamt für Migration und Flüchtlinge, 2013, S. 151 (166); s. auch EuGH, Rs. C-135/08 (Rottmann), Slg. 2010, I-1449, Rn. 45.

82 Ebenso BVerwG, Urteil v. 30. 7. 2013, 1 C $15.12, \mathrm{Rn} .10 \mathrm{ff}$.

83 Zutreffend $M$. Wendel, Aufenthalt (Fn. 69), S. 137.

84 Dies folgt aus dem Rückverweis bei EuGH, Rs. C-34/09 (Ruiz Zambrano), Slg. 2011, I-1177, Rn. 40 auf das Rottmann-Urteil gemäß EuGH, Rs. C-135/08 (Rottmann), Slg. 2010, I-1449, Rn. 51 sowie, a maiorem ad minus, aus den Schranken für mobile Unionsbürger gemäß EuGH, Rs. C-145/09 (Tsakouridis), Slg. 2010, I-11979, Rn. 40 ff.; ausführlicher $K$. Hailbronner/D. Thym, Ruiz Zambrano (Fn. 48), S. 2012 sowie P. Cede in diesem Heft auf S. 79.

85 S. Art. 3 RL 2004/38/EG (Fn. 6) sowie EuGH, Rs. C-256/11 (Dereci u.a.), Slg. 2011, I-11315, Rn. 50-57.

86 Zutreffend BVerwG, Urteil v. 30.7.2013,1 C 15.12, Rn. 9; stattdessen gilt das AufenthG.

87 Vgl. D. Thym, Humanitäres Bleiberecht zum Schutz des Privatlebens?, InfAuslR 2007, S. 133 (137 f.)

88 Näher A. Lansbergen/N. Miller, Citizenship Rights (Fn. 44), S. 298-300. 


\section{Familiennachzug jenseits des Kernbereichs}

Ein Grund, warum drittstaatsangehörige Familienmitglieder rechtlich so viele Probleme verursachen, liegt daran, dass die Mitgliedstaaten ihr klassisches Ausländerrecht zur Anwendung bringen. Eben dieses folgt jedoch einem anderen Grundansatz als die Unionsbürgerschaft. Ausgangspunkt des Ausländerrechts ist nicht das individuelle Recht eines jeden Unionsbürgers auf Grenzübertritt, sondern die prinzipielle Entscheidungshoheit der Staaten über Einreise und Aufenthalt als Ausfluss staatlicher Souveränität. ${ }^{89}$ Dies bedeutet nicht, dass Drittstaatsangehörige keine Rechte besäßen. Im Gegenteil. Heute ist in Europa allgemein anerkannt, dass Ausländer sich auf Grundrechte berufen können. Einzelheiten zu den Grundlagen und Grenzen des Grundrechtsstatus sind umstritten, aber im Grundsatz steht fest, dass speziell Art. 3 und Art. 8 EMRK der staatlichen Entscheidungsfreiheit im Ausländerrecht inhaltliche Grenzen setzen. ${ }^{90}$

Wichtig für die Zwecke des vorliegenden Beitrags ist die Einsicht, dass die Kernbereichsrechtsprechung trotz sachlicher Überschneidungen im Einzelfall von der Anwendung der Grundrechtecharta zu unterscheiden ist (hierzu 1.). Dies gilt umso mehr als die Grundrechte in Migrationsfragen nach der etablierten Rechtsprechung einer anderen Logik folgen als die Privilegien der Unionsbürgerfreizügigkeit (2.). Mit den Grundrechten tritt ein alternativer verfassungsrechtlicher Bezugsrahmen in den Vordergrund, den die Kernbereichsdoktrin zu verstellen drohte: das Migrationsrecht im Raum der Freiheit, der Sicherheit und des Rechts, hinsichtlich dessen das EU-Primärrecht eigenständige Vorgaben tätigt (3.).

\section{Kernbereich und Grundrechtecharta}

Die Abgrenzung zwischen der Kernbereichsdoktrin und den Grundrechten war anfangs unklar, weil der Gerichtshof den sachlichen Inhalt des Kernbereichs nicht näher definiert hatte. Dies führte zur Vermutung, dass jedenfalls der Wesensgehalt der Unionsgrundrechte zugleich dem Kernbereich unterfalle. ${ }^{91}$ Diese Überlegungen sind freilich hinfällig, seit der Gerichtshof die Kernbereichsdoktrin restriktiv gedeutet hat und diese im Kern als Schutzschild gegen eine Pflicht zum Verlassen des Unionsgebiets ausbaute..$^{92}$ Seither steht fest, dass die Kernbereichsdoktrin und die Anwendung der EU-Grundrechte zwei getrennte Themen sind. Nach allgemeinen Regeln gelten die EU-Grundrechte nicht bei reinen Inlandssachverhalten, weil Art. 51 GRC die Mitgliedstaaten ausschließlich bei der Durchführung des Unions-

89 Zur klassischen Sichtweise s. K. Hailbronner/J. Gogolin, Aliens, in: Wolfrum (Hrsg.), The Max Planck Encyclopedia of Public International Law, OUP: online edition, Rn. 14 ff. sowie EGMR, Urteil v. 18.2.1991, Nr. 12313/86 (Moustaquim/Belgien), Rn. 43.

90 Ausführlicher J. Gundel, Der grundrechtliche Status der Ausländer, in: Isensee/Kirchhof(Hrsg.), Handbuch des Staatsrechts, Bd. IX, 3. Aufl. 2011, §198.

$91 \mathrm{Vgl}$. A. von Bogdandy et al., Reverse Solange. Protecting the Essence of Fundamental Rights against EU Member States, CMLRev. 2012, S. 489 (500-507), wobei es nicht um den Familiennachzug ging und auch nicht behauptet wurde, dass nationale Regelungen hierzu den Wesensgehalt des Familiengrundrechts antasteten.

92 S. dazu oben III.2 
rechts an die EU-Grundrechte bindet. Diese Anwendungsschwelle deutet der EuGH in der jüngeren Rechtsprechung großzügig, ${ }^{93}$ unterstellt jedoch weiterhin nur diejenigen Sachverhalte der Charta, die vom Anwendungsbereich des primären oder sekundären Unionsrechts umfasst sind. ${ }^{94}$

Hieraus folgt für unsere Zwecke, dass eine Anwendung des EU-Grundrechts zum Schutz des Familienlebens gemäß Art. 7 GRC, das inhaltlich mit Art. 8 EMRK übereinstimmt, ${ }^{95}$ voraussetzt, dass der zu entscheidende Sachverhalt dem Unionsrecht unterfällt. Diese Anwendungsschwelle kann durch EU-Richtlinien mit Regeln zum Familiennachzug ${ }^{96}$ ebenso eröffnet werden wie durch das Assoziierungsabkommen EWG-Türkei. ${ }^{97}$ Nur soweit dies zutrifft, gelten die EU-Grundrechte, weil die Hürde des Art. 51 GRC überwunden wurde. Das gleiche Ergebnis kann eintreten, wenn die nationalen Gerichte nach Maßgabe der EuGH-Rechtsprechung zu dem Ergebnis gelangen, dass infolge einer Abschiebung der Eltern eine faktische Ausreisepflicht eines jugendlichen Unionsbürgers aus dem Unionsgebiet droht, die ihrerseits die Kernbereichsdoktrin verletzen würde.

Wichtig ist das Bewusstsein für die konditionale Verknüpfung zwischen der Kernbereichs-Rechtsprechung und den EU-Grundrechten. Das Grundrecht auf Privatund Familienleben gemäß Art. 7 GRC gehört für sich genommen nicht zum Kernbestand der Unionsbürgerrechte und findet auf einen Sachverhalt nur Anwendung, wenn dieser aufgrund einer separaten Prüfung entweder der Kernbereichsdoktrin unterfällt oder anderweitig von EU-Recht geregelt wird. ${ }^{98}$ Fällt diese Prüfung positiv aus, gelten die EU-Grundrechte; andernfalls müssen die nationalen Gerichte das nationale Verfassungsrecht sowie die EMRK als Völkerrecht anwenden. ${ }^{99}$

\section{Migrationsrecht und Grundrechte}

Beim Familiennachzug überschneiden sich die Unionsbürgerfreizügigkeit und die Grundrechte, weil sie denselben Lebenssachverhalt regeln. Das bedeutet jedoch nicht, dass beide Rechtskomplexe derselben Logik folgten. Das Gegenteil trifft zu, weil der grundrechtliche Schutz des Privat- und Familienlebens anderen Regeln folgt als die Unionsbürgerrechte unter Einschluss der Kernbereichsdoktrin. Der Unterschied folgt aus den einleitenden Bemerkungen. Seit den 1960er-Jahren schüt-

93 S. EuGH, Urteil v. 26.2.2013, Rs. C-617/10 (Åkerberg Fransson), Rn. $18 \mathrm{ff}$.

94 Hierzu ausführlicher C. Ohler, Grundrechtliche Bindungen der Mitgliedstaaten nach Art. 51 GRCh, NVwZ2013, S. 1433 (1434-1437) und D. Thym, Die Reichweite der EU-Grundrechte-Charta, NVwZ 2013, S. 889 (890 ff.).

95 Vgl. Art. 52 Abs. 3 GRC sowie EuGH, Rs. C-400/10 PPU (McB), Slg. 2010, I-8965, Rn. 53.

96 Im Fall des Familiennachzugs insb. RL 2004/38/EG (Fn. 6) bzw. RL 2003/86/EG (ABI. EU 2003 L 251/12), die freilich einen personalen und sachlichen Anwendungsbereich besitzen, der speziell beim Familiennachzug zu nichtmobilen Unionsbürgern regelmäßig nicht greift; vgl. EuGH, Urteil v. 8.5.2013, Rs. C-87/12 (Ymeraga und Ymeraga-Tafarshiku), Rn. 42; Rs. C-256/11 (Dereci u.a.), Slg. 2011, I-11315, Rn. $46 \mathrm{ff}$.

97 Speziell im Dereci-Urteil stand im Raum, dass ein Sachverhalt, der dem EuGH vorlag, eventuell dem Assoziierungsabkommen unterfälit.

98 Ebenso K. Lenaerts, Court's (Fn. 59), S. 51 f.; M. Wendel, Aufenthalt (Fn. 69), Abschn. IV.1.b; S. Reynolds, Exploring (Fn. 59), S. 382-384; K. Hailbronner, EU-Freizügigkeitsgesetz (Fn. 81), S. 166; sowie P. Mengozzi, Schlussanträge v. 29.11.2011, EuGH, Rs. C-256/11 (Dereci u.a.), Slg. 2011, I-11315, Rn. 37 f.; s. auch P. Cede in diesem Heft auf S. 79.

99 So die Regieanweisung bei EuGH, Rs. C-256/11 (Dereci u.a.), Slg. 2011, I-11315, Rn. 72 f. 
zen der EU-Gesetzgeber und der EuGH den Familiennachzug nahezu bedingungslos. Gemeinwohlbelange unterhalb der Schwelle einer Gefahr für die öffentliche Ordnung, Sicherheit und Gesundheit können dem Zuzug von Familienangehörigen von mobilen Unionsbürgern nicht entgegen gehalten werden. ${ }^{100}$ Eben dies ist bei den Grundrechten anders.

Nach der gefestigten EGMR-Rechtsprechung gewähren die Grundrechte im Regelfall keinen unbedingten Anspruch auf Gebietszugang oder Gebietsverbleib zum Zwecke der Familieneinheit. ${ }^{101}$ Vielmehr sind in einer umfassenden Güterabwägung die privaten Belange der Migranten zu den öffentlichen Interessen des Aufenthaltsstaats in Bezug zu setzen. Speziell für die Ausweisung von Familienangehörigen entwickelte der EGMR acht Kriterien, die ihrerseits die Prüfung der Verhältnismäßigkeit durch nationale Gerichte anleiten sollen. Diese lauten: Schwere des Rechtsverstoßes, Aufenthaltsdauer, Verhalten nach dem Rechtsverstoß, Staatsangehörigkeit der beteiligten Personen, Familiensituation, Kenntnis des Partners vom Rechtsverstoß bei Beziehungsbeginn, Alter der Kinder sowie Hindernisse für eine Umsiedlung in den Herkunftsstaat. ${ }^{102}$ Ergänzt wurden später das Kindeswohl sowie die Festigkeit der gesellschaftlichen, kulturellen und familiären Beziehungen zum Herkunfts- sowie zum Aufenthaltsstaat. ${ }^{103}$ Hierbei spielen auch eventuelle Verstöße gegen das Migrationsrecht eine Rolle, weil der EGMR ganz allgemein dem öffentlichen Interesse strukturell einen breiteren Raum einräumt als der EuGH bei der Unionsbürgerschaft. ${ }^{104}$

Die vorstehenden Ausführungen klingen abstrakt, besitzen jedoch greifbare Folgen. Aus den genannten Kriterien folgt ganz konkret, dass die Vertragsstaaten der EMRK gegenüber Drittstaatsangehörigen eine Vielfalt von Gemeinwohlbelangen verfolgen dürfen, die das Ausländerrecht prägen und die der Unionsgesetzgeber im Rahmen der Unionsbürgerfreizügigkeit für unbeachtlich erklärt. Hierzu drei Beispiele: Erstens verbietet das EU-Recht die Ausweisung von Unionsbürgern aus generalpräventiven Gründen und verlangt nach dem eindeutigen Wortlaut des Sekundärrechts: „Bei Maßnahmen der öffentlichen Ordnung oder Sicherheit darf ausschließlich das persönliche Verhalten der in Betracht kommenden Einzelpersonen ausschlaggebend sein. Strafrechtliche Verurteilungen allein können ohne weiteres diese Maßnahmen nicht begründen." ${ }^{105}$ Auf dieser Grundlage erteilte der EuGH dem deutschen Stufensystem von Ist-, Regel- und Ermessensausweisung eine Absage,

$100 \mathrm{~S}$. dazu oben II.

101 Ausführlicher P. Boeles/M. den Heijer/G. Lodder/K. Wouters, European Migration Law, 2009, S. $141 \mathrm{ff}$. und A. Zimmermann, Ausweisungsschutz, in: Grote/Marauhn (Hrsg.), Konkordanzkommentar GG/EMRK, 2006, Kap. 27, Rn. $82 \mathrm{ff}$.

102 EGMR, Urteil v. 2.8.2001, Nr. 54273/00 (Boultif/Schweiz), Ziff, 48.

103 Vgl. EGMR, Urteil v. 18.10.2006 (GK), Nr. $46410 / 99$ (Üner/Niederlande), Ziff. 58, wobei bei Personen, die den Großteil ihrer Sozialisation im Herkunftsstaat erhielten, besonders streng aufgrund weniger Kriterien zu prüfen ist; vgl. EGMR, Urteil v. 23. 6. 2008 (GK), Nr. 1638/03 (Maslov/Österreich), Ziff. 71.

104 Ausführlicher D. Thym, Residence as de facto Citizenship?, in: Rubio-Marin (Hrsg.), Human Rights and Immigration, 2014, S. $106(121-126)$.

105 Art. 3 Abs. 1 f. RL 64/221/EWG (ABl. EG 1964, S. 850) sowie, heute, Art. 27 Abs. 2 RL 2004/38/EG (Fn. 6). 
das seither auf Unionsbürger nicht mehr angewandt werden darf. ${ }^{106}$ Auch aus den Grundrechten, insbesondere der EMRK, folgen inhaltliche Grenzen für das nationale Ausweisungsrecht, die das deutsche Stufensystem in Frage stellen. Allein diese Grenzen sind weitaus weniger engmaschig. So greifen die EGMR-Standards für den Familiennachzug regelmäßig nur dann, wenn eine Person enge familiäre Beziehungen im Inland aufweist oder als dort verwurzelt gelten darf. ${ }^{107}$ Zudem genießen die öffentlichen Interessen allgemein ein größeres Gewicht und sogar eine Ausweisung aus generalpräventiven Gründen darf, anders als bei Unionsbürgern, speziell nach schweren Straftaten weiterhin verhängt werden. ${ }^{108}$ Auch eine dauerhafte Trennung von Eltern und Kindern ist zulässig. ${ }^{109}$

Zweitens darf der Familiennachzug nach deutschem Recht regelmäßig verweigert werden, wenn der finanzielle Lebensunterhalt nicht gesichert ist. ${ }^{110}$ Beim Familiennachzug zu Unionsbürgern ist dies nicht der Fall - vorausgesetzt der Unionsbürger übt eine Beschäftigung aus, die einen Arbeitnehmerstatus begründet, der seinerseits dem Arbeitnehmer und der Familie einen gleichberechtigten Zugang zu sozialen und steuerlichen Vergünstigungen sichert. ${ }^{111}$ Einzig bei wirtschaftlich inaktiven Unionsbürgern erfordert das Freizügigkeitsrecht ausreichende Existenzmittel, ${ }^{112}$ deren Fehlen den Gerichtshof nicht von der Zuerkennung eines gleichen Sozialleistungszugangs abhielt. ${ }^{113}$ Im Fall der EMRK ist dies anders: Die Vertragsstaaten dürfen den Familiennachzug von Drittstaatsangehörigen regelmäßig verweigern, wenn der finanzielle Lebensunterhalt nicht gesichert ist. ${ }^{114}$ Dies verdeutlicht den großzügigen Zugriff der Unionsbürgerschaft, die den Familiennachzug ganz ähnlich regelt wie im Bundesstaat. ${ }^{115}$

Drittens darf von Unionsbürgern nicht verlangt werden, dass sie die Landessprache erlernen - und zwar von Arbeitnehmern ebenso wenig wie im Anwendungsbereich des Art. 21 AEUV. Ein Deutscher aus Lindau am Bodensee, der ins österreichische Bregenz zieht, hat einen Anspruch auf Familiennachzug für seine Ehefrau aus der Türkei, ohne dass diese Deutsch verstehen muss ${ }^{116}$ - obgleich in derselben Kon-

106 Vgl. EuGH, Rs. $67 / 74$ (Bonsignore), Slg. 1975, 297, Rn. 4 f. sowie EuGH, verb. Rs. C-482/01 u. C-493/01 (Orfanopoulos und Olivieri), Sig. 2004, I-5257, Rn. 62-71.

107 Ausführlicher D. Thym, Migrationsverwaltungsrecht, 2010, S. 228-253 sowie M. Eckertz-Höfer, Neuere Entwicklungen in Gesetzgebung und Rechtsprechung zum Schutz des Privatlebens, ZAR 2008, S. 41-46 u. S. 93-96.

108 So zutreffend BVerwGE 142, 29; s, auch die Billigung einer generalpräventiven Ausweisung durch EGMR, Urteil v. 6.12.2007, Nr. 69735/01 (Chair/Deutschland), Ziff. 24

109 In der jüngeren Vergangenheit EGMR, Urteil v. 15.11.2012, Nr. 38005/07 (Kissiwa Koffi/Schweiz); EGMR, Urteil v. 15.5.2012, Nr. $16567 / 10$ (Nacic u.a./Schweden); EGMR, Urteil v. 10.5.2012, Nr. 45237/08 (Madah u.a./Bulgarien); EGMR, Urteil v. 14.2.2012, Nr. 26940/10 (Antwi u.a./Norwegen); sowie in Bezug auf Deutschland EGMR, Urteil v. 6.12.2007, Nr. 69735/01 (Chair/Deutschland, Ziff. 66.

110 Vgl. die allgemeine Erteilungsvoraussetzung gemäß $\$ 5$ Abs. 1 Nr. 1 AufenthG.

111 Näher hierzu Fn. 11 und begleitender Text.

112 So Art. 7 Abs. 1 lit. b RL 2004/38/EG (Fn. 6).

113 Ausführlicher R. Rebhahn in diesem Heft auf S. 95.

114 S. EGMR, Urteil v. 26.4.2013, Nr. $16351 / 03$ (Konstatinov/Niederlande), Ziff. 50 sowie BVerwG, Urteil v. $30.4 .2009,1$ C 3.08 , Rn. $13 \mathrm{ff}$

115 Zur Vergleichbarkeit der EU-Regeln mit der Bundesstaatsentwicklung im Bereich des Sozialrechts s. C. Schönberger, Unionsbürger, $2005, \S 19$.

116 Hierzu bereits oben Fn. 15 und begleitender Text. 
stellation der Familiennachzug regelmäßig verweigert würde, wenn der Deutsche in Lindau wohnen bliebe, weil Deutschland den Familiennachzug zu Deutschen und Ausländern in bestimmten Konstellationen regelmäßig verweigert, wenn keine einfachen Kenntnisse der deutschen Sprache vorliegen. ${ }^{117}$ Eben dies verstößt nach der Einschätzung deutscher sowie britischer Gerichte nicht gegen Art. $8 \mathrm{EMRK}^{118}$ und es ist davon auszugehen, dass der EuGH und der EGMR diese Rechtsauffassung in der Zukunft bestätigen werden. ${ }^{119}$

\section{Migrationsrecht}

Aus den vorstehenden Ausführungen folgt, dass zwischen Unionsbürgerschaft und Menschenrechten unterschieden werden muss. Es macht einen gravierenden Unterschied, ob wir den Familiennachzug nach den großzügigen Regeln der Unionsbürgerfreizügigkeit oder den Grundrechten beurteilen. Aus diesem Grund ist es wichtig, dass der EuGH im Dereci-Urteil auf die Grundrechte als alternativen Prüfmaßstab neben der Kernbereichsdoktrin verweist. ${ }^{120}$ Und aus demselben Grund ist es wichtig, dass dasselbe Urteil sowie die Entscheidungen Iida und $O$. und $S$. hervorheben, dass es zwei verschiedene Blickwinkel auf den Familiennachzug gibt: Neben die Unionsbürgerschaft tritt die Harmonisierung des nationalen Ausländerrechts durch den EU-Gesetzgeber, in den genannten Fällen durch die Familiennachzugs-Richtlinie 2003/86/EG bzw. die Daueraufenthalts-Richtlinie 2003/109/ EG. ${ }^{121}$ Damit anerkennt der EuGH, dass das Migrationsrecht einen alternativen verfassungsrechtlichen Referenzrahmen für die Beurteilung des Familiennachzugs neben der Unionsbürgerschaft bereitstellt. ${ }^{122}$

Aus der Perspektive des Primärrechts sind die genannten Richtlinien zum Familiennachzug sowie dem Daueraufenthalt allerdings nicht Ausfluss der Unionsbürgerschaft. Sie unterfallen stattdessen dem EU-Migrationsrecht, das der EuGH mit dem Fokus auf die Unionsbürgerschaft aus den Augen zu verlieren drohte. Eben dies folgt rechtlich und konzeptionell jedoch gerade nicht der Logik der Unionsbürgerrechte. An die Stelle des Binnenmarkts und der Unionsbürgerschaft, die beide auf die Maximierung der transnationalen Freiheit von Einzelpersonen im Dienste der EU-Integration ausgerichtet sind, tritt der Raum der Freiheit, der Sicherheit und

117 Vgl. \$30 Abs. 1 S. 1 Nr. 2 i.V.m. \& 28 Abs. 1 S. 5 AufenthG.

118 I.d.S. insb. BVerwGE 136, 231; BVerfG, Beschluss v. 25.3.2011, 2 BvR 1413/10; BVerwG, Urteil v. 4.9.2012, 10 C 12.12; und UK Court of Appeal, Urteil v. 12.4.2013 (Bibi v SSHD), [2013] EWCA Civ 322; die allerdings eine Ausnahme (Härtefallklausel) verlangen.

119 Beim EuGH ist zur Auslegung der Richtlinie das Verfahren $K$. und $A$. anhängig (Rs. C-153/14); die Bedeutung von Sprachkenntnissen für die Güterabwägung i.R.d. Art. 8 EMRK bekräftigt EGMR, Urteil v. 14.2.2012, Nr. 26940/10 (Antwi u.a./Norwegen), Ziff. 94.

120 S. EuGH, Rs. C-256/11 (Dereci u.a.), Slg. 2011, I-11315, Rn. 70-73 sowie D. Thym, Anmerkung, NVwZ 2012, S. 1003 (1004).

121 Auf das Migrationsrecht neben den Unionsbürgerrechten verweist EuGH, Rs. C-256/11 (Dereci u.a.), Slg. 2011, I-11315, Rn. 44 ff.; Urteil v. 8.11.2012, Rs. C-40/11 (Iida), Rn. 36-48; Urteil v. 6.12.2012, verb. Rs. C-356/11 u. C-357/11 (O. und S.), Rn. $61 \mathrm{ff}$.

122 Sinngemäß A. Lansbergen/N. Miller, Citizenship Rights (Fn. 44), S. 300 f.; D. Thym, 'Real' Citizenship (Fn. 54), S. 170; sowie A. Tryfonidou, Outer Boundaries (Fn. 63), S. 524. 
des Rechts, der dem EU-Gesetzgeber sowie nationalen Parlamenten erweiterte Gestaltungsspielräume bietet. Wegweisend bestimmt Art. 79 Abs. 1 AEUV: „Die Union entwickelt eine gemeinsame Einwanderungspolitik, die in allen Phasen eine wirksame Steuerung der Migrationsströme... gewährleisten soll.“

Wichtig ist das Bewusstsein für die inhaltliche Offenheit dieses primärrechtlichen Steuerungsanspruchs. Dieser enthält keine direkte Vorgabe für eine großzügige oder restriktive Politikgestaltung. ${ }^{123}$ Die Entscheidung zwischen Abschottung und Grenzöffnung erfolgt - im Rahmen der Grundrechte - im Gesetzgebungsverfahren. Diese Offenheit für politische Entscheidungen ist der zentrale Unterschied zum Freizügigkeitsregime des Binnenmarkts. ${ }^{124}$ Drittstaatsangehörige profitieren von keinen Wanderungsrechten mit Verfassungsrang nach dem Modell der Unionsbürgerrechte, sondern können sich ,nur" auf Grundrechte berufen, die einen geringeren Schutzumfang besitzen. ${ }^{125}$

Beim Familiennachzug zu Unionsbürgern verschwimmen diese beiden Rechtsregime zwangsläufig, weil die „,abgeleiteten“ Unionsbürgerrechte der Familienangehörigen mit den „eigenen“ Grundrechten der Familienmitglieder inhaltlich zusammenfallen. Diese Überschneidung der beiden Rechtsregime bei der Beurteilung eines Sachverhalts ist nichts Neues und wird auch fortbestehen. ${ }^{126}$ Wichtig ist jedoch, dass die Grauzonen der Überschneidung und Überlappung nicht mit einer materiellrechtlichen Einheit verwechselt werden. Hinsichtlich des verfassungsrechtlichen Rahmens muss klar zwischen Unionsbürgerschaft und Migrationsrecht unterschieden werden - und zwar gerade deshalb, weil die Abgrenzung bei konkreten Sachverhalten nicht immer trennscharf möglich ist. Dies gibt der Diskussion inhaltliche Klarheit und der Rechtspraxis eine Orientierung.

Die Unionsbürgerschaft mit der Freizügigkeit und dem Kernbereichsschutz auf der einen Seite sowie das Migrationsrecht mit dem Steuerungsanspruch und den Menschenrechten auf der anderen Seite sind alternative Bezugsrahmen, deren Eigengesetzlichkeit auch der EuGH in der jüngeren Rechtsprechung anerkennt. Der Wissenschaft bleibt die Aufgabe, die dogmatische Abstufung zwischen den beiden Rechtsregimen auch theoretisch in den Griff zu bekommen. Der jeweils eigene $\mathrm{Zu}-$ griff der unterschiedlichen Bezugsrahmen erfordert einen konzeptionellen Überbau, den ich an anderer Stelle als kosmopolitische Grundierung des Migrationsrechts in Abgrenzung zur Unionsbürgerschaft vorgeschlagen habe. ${ }^{127}$

123 Zur fehlenden Determinationskraft D. Thym, in: Grabitz/Hilf/Nettesheim (Hrsg.), Das Recht der Europäischen Union, 2010, Art. 79 AEUV, Rn. 14-17.

124 Hierzu bereits D. Thym, Freizügigkeit (Fn. 22), S. 495-502 sowie D. Thym, EU Migration Policy and its Constitutional Rationale, CMLRev. 2013, S. 709 (721-723).

125 Ausführlicher D. Thym, EU Migration Policy (Fn. 124), S. 718-721.

126 Allgemein zur Überschneidung D. Thym, EU Migration Policy (Fn. 124), S. 714-716.

127 Hierzu D. Thym, EU Migration Policy (Fn. 124), S. 725 ff. sowie D. Thym, Migrationssteuerung im öffentlichen Interesse, in: Jochum et al. (Fn. 58), S. 245-262. 


\section{Fazit}

In quantitativer Hinsicht ist der Familiennachzug seit Jahrzehnten einer der wichtigsten Migrationskanäle. Aus diesem Grund besitzen die Regeln über die Familienzusammenführung eine zentrale Bedeutung für die Migrationspolitik und sorgten zuletzt für Unsicherheit, soweit es um Familienmitglieder von Unionsbürgern aus Drittstaaten geht. Dies ist kein Zufall, weil sich in dieser Situation zwei Rechtsregime überschneiden, die den Familiennachzug unterschiedlich regeln. Zum einen geht es um die Unionsbürgerrechte auf Grundlage der Arbeitnehmerfreizügigkeit, hinsichtlich derer der EU-Gesetzgeber sowie der EuGH seit den 1960er-Jahren den Familiennachzug großzügig ausgestalteten und nahezu keinen inhaltlichen Schranken unterwarfen. Zwar können sich Familienmitglieder aus Drittstaaten nicht direkt auf die Unionsbürgerschaft berufen, sie profitieren jedoch von abgeleiteten Rechten, die eine weitgehende Gleichstellung mit Unionsbürgern bewirken. Zum anderen regeln das EU-Migrationsrecht sowie die Grundrechte in der EMRK sowie der GRC den Familiennachzug aus Drittstaaten und räumen hierbei den öffentlichen Interessen breiteren Raum ein, wenn die Mitgliedstaaten den Familiennachzug etwa von einer finanziellen Sicherung des Lebensunterhalts oder einfachen Sprachkenntnissen abhängig machen dürfen.

Eine neue Komplexität erreichte die Rechtslage, als der EuGH im Urteil Ruiz Zambrano die Familieneinheit von jugendlichen Unionsbürgern unter Berufung auf einen vermeintlichen Kernbestand der Unionsbürgerrechte schützte, ohne dass eine Beschränkung des Freizügigkeitsrechts zu befürchten stand. Die Unionsbürgerschaft schien eine Sogwirkung zu entfalten und den Familiennachzug zu Unionsbürgern umfassend zu regeln. Dieser Paradigmenwechsel blieb jedoch aus, weil der EuGH in Folgeurteilen die Kernbereichsdoktrin einfing und im Kern auf Situationen beschränkte, in denen jugendlichen Unionsbürgern - nicht jedoch Ehepartnern - die faktische Ausreisepflicht aus dem Unionsgebiet droht. Einzelheiten dieser Vorgabe bleiben bis heute unklar und werden hinsichtlich der innerstaatlichen Anwendung in diesem Beitrag diskutiert. Fest steht jedenfalls, dass die Kernbereichsdoktrin auf Sonderfälle beschränkt bleibt. Dies ist zu begrüßen, zumal hierdurch der Blick frei wird auf die speziellen Normierungen im Primär- und Sekundärrecht zum Familiennachzug, die als Bestandteil des EU-Migrationsrechts im Raum der Freiheit, der Sicherheit und des Rechts einer eigenen verfassungsrechtlichen Logik folgen. Dieser unterschiedliche Rechtsrahmen muss konzeptionell auch dann klar von der Unionsbürgerschaft unterschieden werden, wenn die Abgrenzung in der Praxis nicht immer kar ist. 